\title{
Strategies to Reduce the Immunogenicity of Recombinant Immunotoxins
}

\author{
Ronit Mazor, Emily M. King, and Ira Pastan
}

From the Laboratory of Molecular Biology, Center for Cancer Research, National Cancer Institute, National Institutes of Health, Bethesda, Maryland

Accepted for publication

April 6, 2018.

Address correspondence to Ira Pastan, M.D., Laboratory of Molecular Biology, National Cancer Institute, 37 Convent Dr, Room 5106, Bethesda, MD 20892-4264. E-mail: pastani@ mail.nih.gov.

\begin{abstract}
Recombinant immunotoxins (RITs) are genetically engineered proteins being developed to treat cancer. They are composed of an Fv that targets a cancer antigen and a fragment of a bacterial toxin that kills tumor cells. Because the toxin is a foreign protein, it is immunogenic. The clinical success of RITs in patients with a normal immune system is limited by their immunogenicity. In this review, we discuss our progress in therapeutic protein deimmunization and the balancing act between immunogenicity and therapeutic potency. One approach is to prevent the activation of B cells by mapping and elimination of B-cell epitopes. A second approach is to prevent helper T-cell activation by interfering with major histocompatibility complex II presentation or T-cell recognition. Immunizing mice with RITs that were deimmunized by elimination of the murine B- or T-cell epitopes showed that both approaches are effective. Another approach to control immunogenicity is to modify the host immune system. Nanoparticles containing synthetic vaccine particles encapsulating rapamycin can induce immune tolerance and prevent anti-drug antibody formation. This treatment restores RIT anti-tumor activity that is otherwise neutralized because of immunogenicity. (Am J Pathol 2018, 188: 1736-1743; https://doi.org/10.1016/j.ajpath.2018.04.016)
\end{abstract}

Recombinant immunotoxins (RITs) are genetically engineered proteins that are designed to treat cancer. They contain an antibody fragment that targets tumor antigens and a protein toxin that kills the cell. We have been developing RITs that use a $38-\mathrm{kDa}$ fragment of Pseudomonas exotoxin A (PE38) as a payload ${ }^{1}$ (Figure 1A). In chemotherapy-resistant hairy cell leukemia patients, whose immune systems are suppressed by the cancer, RIT treatment produced many complete regressions and prolonged life. $^{2,3}$ In patients with normal immune systems, RITs are immunogenic because of the bacterial origin of the toxin. Anti-drug antibody (ADA) responses were found in $100 \%$ of the mesothelioma patients who were treated with a RIT that targets mesothelin. ${ }^{4,5}$ The ADA neutralized the RIT, accelerated its clearance from the blood, and prevented further treatment. ADAs have a risk of immune-related adverse events, including infusion-related reactions, allergic reactions, anaphylaxis, delayed hypersensitivity, and autoimmunity. ${ }^{6}$ Of the hundreds of patients treated with PE38-based RITs, only a single anaphylactic reaction was reported and occurred immediately after the first infusion of the immunotoxin. ${ }^{4}$ Other trials reported grade 1,2 , or 3 skin reactions that were easily managed by steroids. On the basis of the observation that immunocompromised hematological patients do not mount a strong ADA response, a combination therapy of a RIT with an aggressive immune suppression regimen (cyclophosphamide and pentostatin) was evaluated in 11 patients with advanced chemoresistant mesothelioma. The formation of ADA was delayed so that more cycles could be given, and 4 of 10 patients had dramatic tumor responses that substantially increased survival. ${ }^{7}$ This trial demonstrates that RITs can induce major regressions in

\footnotetext{
Supported by the Intramural Research Program of the Center for Cancer Research, National Cancer Institute, NIH.

Disclosures: None declared.

"Immunotoxin therapy of leukemia and mesothelioma: from concept to clinical reality" was presented by I.P. as the keynote lecture at the 2015 Pathobiology for Investigators, Students, and Academicians meeting on October 9, 2015, Baltimore, MD.

Current address of R.M., MedImmune, Gaithersburg, MD
} 
A

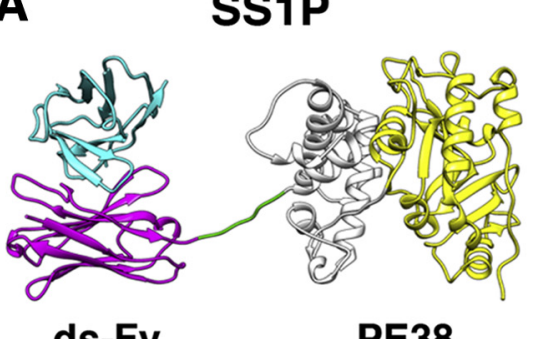

ds-Fv

PE38

C SS1-L010R

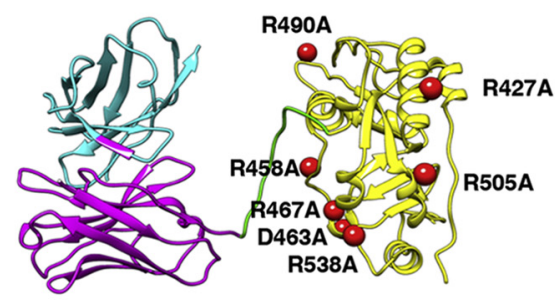

ds-Fv

PE24

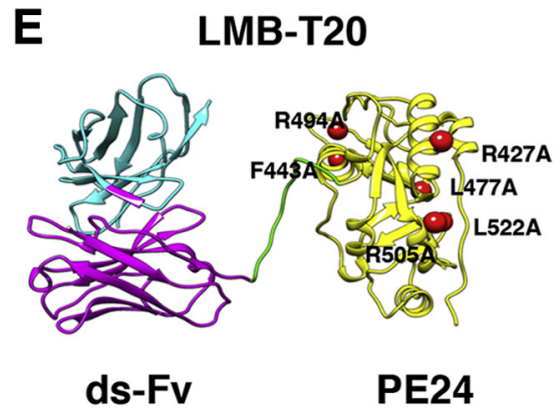

B

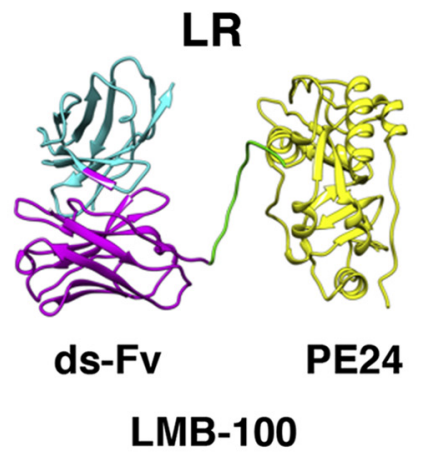

D

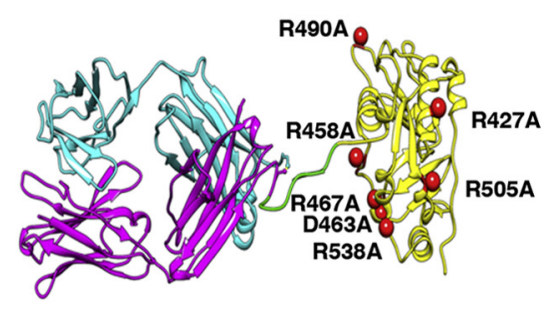

hFab

PE24

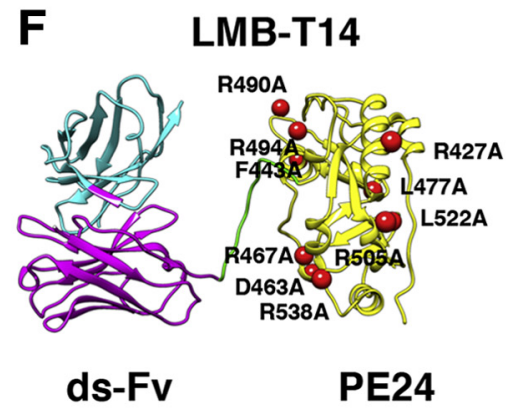

Figure 1 Structural models of recombinant immunotoxins. A: SS1P consists of the disulfidestabilized (ds) heavy chain $\mathrm{Fv}\left(\mathrm{V}_{\mathrm{H}}\right.$; magenta) and light chain $\mathrm{Fv}\left(\mathrm{V}_{\mathrm{L}}\right.$; cyan) of the antibody SS1P. The $V_{H}$ is linked to a $38-k D a$ fragment of Pseudomonas exotoxin A (PE38) that is divided into domain II (gray), domain III (yellow), and part of domain Ib from native PE38. B: Lysosome resistance (LR) immunotoxin. The ds-Fv of SS1P is linked to a 24-kDa fragment consisting of domain III of PE38. C: SS1-L010R. A 24-kDa fragment of PE24 with six point mutations in domain III designed to eliminate binding to B-cell receptors. Point mutations are marked with red balls. D: LMB-100 consists of a humanized Fab linked to the deimmunized L010R PE24 toxin fragment. E: LMB-T20. PE24 with six point mutations in domain III designed to diminish T-cell epitopes. F: LMB-T14. PE24 with 10 point mutations in domain III designed to diminish B- and T-cell epitopes. All models are hypothetical arrangements based on the structures of native PE and IgG; they do not represent actual structure determinations. mesothelioma once immunogenicity is resolved and highlights the need to control immunogenicity to make therapy more effective.

Approaches to control the immunogenicity of therapeutic monoclonal antibodies are based on increasing the human sequence content and include framework humanization, chimerization, and use of mice with humanized germlines. These approaches have been shown to dramatically reduce the rate of anti-mouse and anti-chimera antibodies. ${ }^{8}$ However, some immunogenicity against the variable complementarity-determining region domains may still cause immunogenicity. ${ }^{9}$ Other approaches to mitigate immunogenicity of therapeutic proteins include PEGylation that masks the immunogenic epitopes or combination therapy with immune suppressive molecules that can be toxic and limit the treatment. This review describes our approaches to make less immunogenic RITs by identifying and removing regions of the toxin that are recognized by the immune system as B- and T-cell epitopes. Also, we describe recent experiments that use rapamycin-containing nanoparticles to tolerize the host and prevent ADA formation.

\section{Deletion of Domain II}

Pseudomonas exotoxin A contains three structural domains. Domain I binds to a cellular receptor, domain II is required for processing, and domain III is the catalytic domain. ${ }^{10}$ For immunotoxin generation, domain $\mathrm{I}$ is replaced with an $\mathrm{Fv}$ (Figure 1A) ${ }^{11}$ connected to a $38-\mathrm{kDa}$ toxin fragment (PE38). Weldon et $\mathrm{al}^{12}$ found that domain II is rich in protease cleavage sites. To reduce the immunogenicity of PE38, residues 251 to 394 were removed and replaced by amino acids 274 to 284, which contain a furin cleavage site lysosome resistance (Figure 1B). Deletion of the protease cleavage sites in domain II reduces the processing of the RIT by antigen-presenting cells and consequently reduces antigen presentation to the immune system. The furin cleavage site cannot be removed; it is critical for the release of the toxin from the antibody in the endosomal compartment, which is necessary for cellular intoxication. ${ }^{10}$ These mutant immunotoxins have high cytotoxic activity on most cell lines investigated and decreased ADA formation in mice. ${ }^{13}$ 


\section{Identification of B-Cell Epitopes}

B-cell epitopes are regions on the surface of a protein that bind to B-cell receptors and secreted Igs. In theory, any region on the surface of a protein is a potential B-cell epitope. $^{14,15}$ However, some epitopes clustered on the surface of the antigen can control most of the immune response. ${ }^{16}$ Furthermore, elimination of epitopes that contribute to antibody-mediated neutralization can be effective in suppressing the immune response. ${ }^{17}$

\section{Identification of Conformational B-Cell Epitopes}

Because ADA neutralizes the activity of antigens by binding to surface residues, Onda et $\mathrm{a}^{18}$ mapped the murine surface (conformational) B-cell epitopes in PE38 using monoclonal antibodies made in mice. Later, the human B-cell epitopes in domain III were mapped with the use of Fvs isolated from $B$ cells of patients who were treated with immunotoxins. The strategy was based on the assumption that B-cell epitopes are made up of large amino acids, such as Arg, Glu, and Gln, and that mutating these to Ala will prevent binding to antigen receptors on B cells. ${ }^{19}$ For the human studies, analysis was limited to domain III, because domain II is not present on second-generation immunotoxins ${ }^{12}$ (Figure 1A). We identified seven conformational murine B-cell epitopes in domains II and III and six human epitopes in domain III. Seven point mutations were incorporated in domain III to abrogate these epitopes (Figure 1C).

\section{B-Cell Epitope Elimination Proof of Concept}

To determine whether elimination of the B-cell epitopes is sufficient to prevent ADA formation, Onda et $\mathrm{al}^{20}$ generated a RIT, SS1-LO10R, with a deletion of domain II and with eight point mutations in domain III designed to prevent binding of mouse B-cell receptors to conformational B-cell epitopes on the RIT surface. We found that repeated immunization of mice with mutant eight point mutations induced only a low ADA titer compared with wild-type RIT and did not compromise RIT anti-tumor activity (Figure 2A).

\section{LMB-100}

On the basis of the promising ADA reduction in mice, we collaborated with Roche (Basel, Switzerland) to develop a new immunotoxin against mesothelin named LMB-100 (alias RG7787). LMB-100 (Figure 1D) has a humanized Fab version of the SS1 mouse Fv linked to a toxin that has a deletion of most of domain $\mathrm{II}^{10,12}$ and seven mutations in domain III (R490A, R456A, R427A，R505A，R467A, D463A, and R538A). ${ }^{23,24}$ LMB-100 shows excellent anti-tumor activity in animal models. ${ }^{25,26}$ Clinical testing for LMB-100 started late in 2014. Preliminary observations in
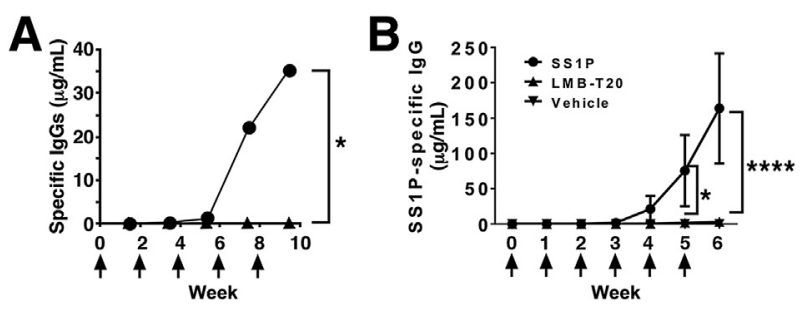

Figure 2 Successful deimmunization of immunotoxins in mice. A: Immunogenicity of HA22 versus HA22-LR-8M in mice. Groups of BALB/C mice received i.v. HA22 $(250 \mu \mathrm{g} / \mathrm{kg}$; circle) or HA22-LR-8M $(500 \mu \mathrm{g} / \mathrm{kg}$; triangle; arrows) and were bled 10 days after each injection. Adapted and modified from Onda et $\mathrm{al}^{21}$ with permission from the Proceedings of the National Academy of Science of the United States. B: Immunization with LMB-T20 impedes formation of antigen-specific IgG. BALB/c mice were immunized with SS1P, LMB-T20, or phosphate-buffered saline (arrows). Serum samples were taken 2 days before every immunization. Antigen-specific antibody titer was evaluated using direct enzyme-linked immunosorbent assay. Adapted and modified from Mazor et $\mathrm{al}^{22}$ with permission from Springer Nature. $n=9(\mathbf{A}) .{ }^{*} P<0.05,{ }^{*} * * P<0.0001$.

patients treated with LMB-100 show that ADA formation is delayed but not abolished (I.P., unpublished data).

\section{Identification of T-Cell Epitopes}

Although elimination of all B-cell epitopes from a molecule is challenging because of the adaptive nature of B-cell specificities and their ability to undergo affinity maturation, ${ }^{14,15}$ T-cell receptor specificity does not change on antigen encounter. Once T-cell epitopes are identified, formation of new specificities is not expected.

\section{T-Cell Proof of Concept in Mice}

Elimination of T-cell epitopes is now considered a wellaccepted strategy to deimmunize protein therapeutics. ${ }^{27-31}$ Yeung et $\mathrm{al}^{32}$ showed that elimination of T-cell epitopes in interferon- $\beta$ resulted in elimination of the ADA response in $\mathrm{BALB} / \mathrm{c}$ mice. To test if elimination of T-cell epitopes can prevent ADA formation to RITs, which represent a high immunologic challenge because of the bacterial origin of the toxin, we mapped murine T-cell epitopes in PE38. Mice were immunized with RIT, and the recovered splenocytes were restimulated with an overlapping peptide library spanning the sequence of PE38. T-cell activation was assayed using IL-2 enzyme-linked immunospot assay. ${ }^{22} \mathrm{We}$ found that $\mathrm{BALB} / \mathrm{c}$ mice have one major and one minor T-cell epitope that correspond to two human T-cell epitopes (amino acids 290 to 308 and 436 to 453 , respectively). Those epitopes are eliminated by deleting domain II and introducing a point mutation in domain III that prevents Tcell activation. We found that repeated immunization of naïve and sensitized mice with the T-cell deimmunized variant induced a low ADA titer that did not neutralize the RIT and did not compromise anti-tumor activity, indicating that this approach is effective (Figure 2B). 


\section{Analysis of HLA Binding Prediction to Identify T-Cell Epitopes}

To identify the location of human T-cell epitopes in PE38, we performed and compared computational-based human leukocyte antigen (HLA) binding analysis with experimental T-cell epitope mapping. HLA binding and presentation is a primary requirement for T-cell activation. Algorithms that predict the affinity of peptides to polymorphic HLA II molecules are commonly used to predict potential T-cell epitopes. ${ }^{33,34}$ To identify regions in PE38 that have a high likelihood to bind to HLA II and consequently be T-cell epitopes, we used two primary HLA binding algorithms (Propred ${ }^{35}$ and IEDB consensus ${ }^{36}$ ). We analyzed multiple thresholds and analysis parameters to identify peptides in PE38 that are predicted to bind with high affinity to multiple HLA II molecules. ${ }^{37}$ Each peptide with a percentile rank $\geq 6 \%$ was considered positive with analysis of 15 HLA-DR alleles. The top nine predicted binders were in amino acids 285 to 311 and 306 to 323 in domain II and 415 to 432,430 to 450,448 to 462,460 to 483,487 to 516,508 to 525 , and 526 to 540 in domain III.

\section{Experimental T-Cell Epitope Mapping}

To identify T-cell epitopes experimentally, we used peripheral blood mononuclear cells from 50 naïve donors and 16 immunized patients that represent the HLA distribution of the patient population in the United States and Europe. Peripheral blood mononuclear cell samples were expanded in vitro for 14 days with the entire RIT and stimulated with a 15-mer peptide library with an overlap of 12 amino acids spanning the sequence of PE38. ${ }^{38,39}$ The addition of an in vitro expansion step was based on the work from the laboratory of Sette and colleagues ${ }^{40}$ and improved the sensitivity of the assay to allow us to detect low abundant $\mathrm{T}$ cells that could not be detected without this step. Furthermore, in vitro expansion before T-cell epitope mapping improves the specificity because it eliminates activation by irrelevant peptides that are not processed to form functional peptides. We identified eight major T-cell epitopes (267 to 281, 276 to 290, and 294 to 308 in domain II and 419 to 432,436 to 453 , 466 to 480,493 to 510 , and 544 to 561 in domain III) (Figure 1E).

Of interest, the two murine T-cell epitopes overlap with two human ones. BALB/c mice are inbred and only have two major histocompatibility complex II presentation molecules (IA and IE), which result in a uniform and minimal epitope map. Human HLA II molecules, on the other hand, are extremely polymorphic, with three (potentially heterozygous) presentation molecules, which results in diverse responses to multiple peptides and a higher total number of promiscuous epitopes. ${ }^{41}$
Comparison of Predicted HLA Binding and Experimental T-Cell Epitopes

To evaluate if predicted HLA II binding affinity matched the experimentally determined T-cell epitopes, we compared the top nine experimental T-cell epitopes with the top nine predicted epitopes. Of the top nine epitopes of each method, five epitopes overlap. ${ }^{37}$ Four epitopes were overpredicted, and four epitopes were underpredicted. Analysis of the epitopes that were underpredicted revealed that some of the epitopes would have been predicted positive if the threshold had been loosened (meaning, if we looked for the top 20 or 30 epitopes instead of the top nine). However, one epitope was completely overlooked by the algorithm. Overpredictions are expected because of the multiple steps in T-cell activation that cannot be predicted by the algorithm, including endosomal processing, T-cell recognition, and $\mathrm{T}$-cell activation. Underpredictions, on the other hand, are not expected, and the existence of true false negatives (ones that would not be predicted to bind to HLA class II molecules, even in low thresholds) undermines the effectiveness of current computational tools for T-cell epitope predictions.

\section{False-Negative T-Cell Epitope Prediction Is Attributable to Non-DR Presentation}

T-cell epitope prediction using HLA binding algorithms focuses on DR molecules and rarely on DP or DQ..$^{30,31,42-46}$ This trend is, in part, because of an assumption that DP molecules are less important for immune responses than DR molecules ${ }^{47}$ and because cell surface expression of DP and DQ is lower than that of DR on resting antigen-presenting cells. ${ }^{48-50}$ This lack of emphasis on DP and DQ alleles led to smaller data sets, which affects the accuracy of peptide binding affinity predictions for these alleles.

To determine how peptides from PE38 are presented, we used an antibody HLA inhibition assay and found that DR restricted only $65 \%$ of the responses, whereas DP and DQ restrict the other $35 \%$ of responses. ${ }^{51}$ One epitope that was undetected using a binding prediction algorithm was $100 \%$ restricted to DP. The algorithm could not have predicted binding to this peptide because of the DR query settings. Of importance, additional prediction analysis that included DP alleles in the analysis still failed to recognize this epitope as a strong binder. This indicates that HLA binding algorithms are not sufficient to accurately predict some T-cell epitopes. However, they do a better job at predicting DR restricted epitopes than DP. At the current time, an experimental approach, such as T-cell activation, is required.

\section{Rational Design of T-Cell-Deimmunized RITs}

The eight human T-cell epitopes were then analyzed to identify point mutations that can diminish T-cell reactivity and yet not compromise RIT cytotoxic activity. We 

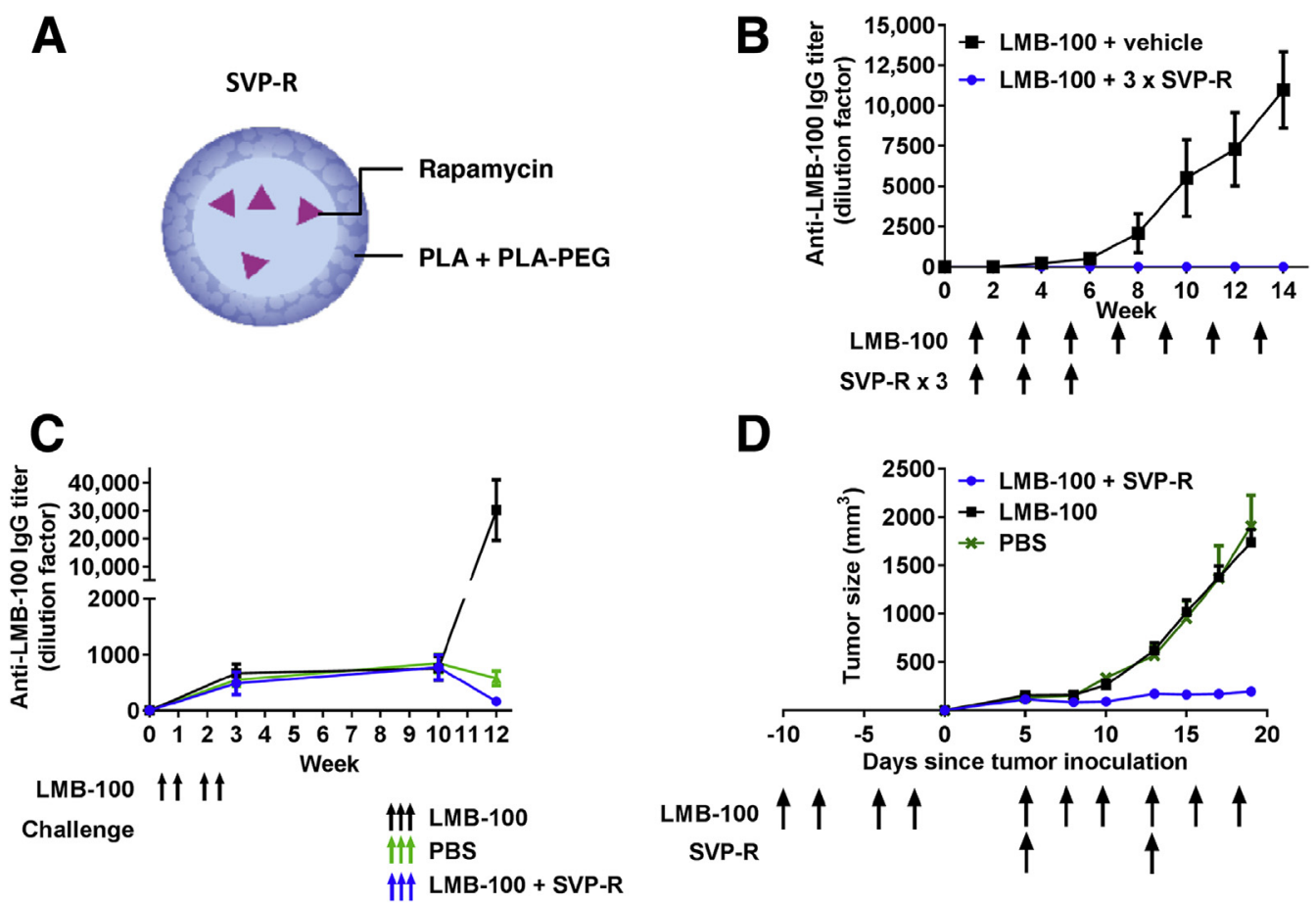

Figure 3 Synthetic vaccine particles encapsulating rapamycin (SVP-R) prevent anti-drug antibody (ADA) response against LMB-100 and restore the neutralized anti-tumor activity. A: Schematic of SVP-R. B: Suppression of ADA with SVP-R. Mice were injected intravenously with LMB-100 + vehicle or LMB-100 + SVP-R once per week for 3 weeks. Mice were then challenged with LMB-100 alone (arrows). Blood samples were collected weekly, and plasma anti-LMB-100 antibodies were detected by enzyme-linked immunosorbent assay (ELISA). C: Anti-tumor efficacy of LMB-100 is restored by addition of SVP-R. Mice were immunized with LMB-100 before tumor inoculation (black arrows). Tumors were then treated with phosphate-buffered saline (PBS; green arrows), LMB-100 (black arrows), or LMB-100 + SVP-R (blue arrows). D: Mice were immunized with LMB-100 and rested for 6 weeks. Mice were then challenged with LMB-100, PBS, or LMB-100 + SVP-R (arrows), and anti-LMB-100 antibodies were measured by ELISA. Adapted and modified from Mazor et al, ${ }^{57}$ with permission from the Proceedings of the National Academy of Science of the United States. PEG, polyethylene glycol; PLA, polylactic acid.

synthesized alanine mutant peptides and screened all responsive donors to each epitope with the mutant peptides. ${ }^{39}$ After identifying several mutations that reduced T-cell activation in most samples, we generated corresponding mutant RITs to test the effect of the mutations on cytotoxic activity. LMB-T18 and LMB-T20 are two T-cell-deimmunized RITs that target CD22 and mesothelin, respectively, which were evaluated for T-cell reactivity and anti-tumor efficacy. ${ }^{39,52}$ Both were engineered to have six point mutations in domain III (R427A, F443A, L477H, R494A, R505A, and L552E) and a major deletion of amino acids 251 to 273 and 285 to 394 from domain II. ${ }^{12}$ Stimulation of peripheral blood mononuclear cells with the full-length LMB-T20 protein induced a weak T-cell activation, dramatically lower than that of the parent RIT, SS1P. ${ }^{52}$ This indicates that elimination of the T-cell epitopes in PE38 was effective in preventing T-cell activation to the whole protein.

LMB-T18 and LMB-T20 have improved cytotoxic activity and anti-tumor activity in cell lines, patient-derived tumor cells, xenograft mice, and patient-derived xenograft mice. ${ }^{39,52}$

The T-cell-deimmunized toxin was also used to generate an immunotoxin that targets glypican 3 for liver cancer treatment. ${ }^{53}$ It has high cytotoxicity on liver cancer cell lines as well as good anti-tumor activity in mice.
Interestingly, when the toxin in LMB-T20 was linked to an $\mathrm{Fv}$ that targets human $\mathrm{CD} 25$ to kill human T-cell malignancies, we found that deletion of domain II significantly impaired the cytotoxic activity. ${ }^{54,55}$ The dependency in domain II for cytotoxic activity is receptor specific and may be attributable to a variable internalization pathway for CD25-mediated killing. To better kill CD25-expressing cells and decrease immunogenicity, we retained domain II and deimmunized it by introducing three point mutations into domain II (E270A, L299A, and Q283A). ${ }^{54}$

\section{Combination of B- and T-Cell Epitopes}

Theoretically, elimination of all of the T-cell epitopes should prevent T-cell help, prevent B-cell affinity maturation and class switching, and prevent formation of neutralizing antibodies. Similarly, elimination of all B-cell epitopes should prevent the initial B-cell recognition and avoid the entire immune response. It has been possible to identify and silence, by mutation, all B- and T-cell epitopes in Balb/c mice. However, it has not been possible to identify all of the human T-cell epitopes or make mutant immunotoxins with all epitopes completely suppressed in humans because human major histocompatibility complex class II molecules are highly polymorphic. The B- and 
T-cell epitope mapping described above identified the strongest and promiscuous epitopes.

To maximize the deimmunization of RITs, we incorporated the mutations that eliminated $\mathrm{T}$ - and $\mathrm{B}$-cell epitopes into a final RIT that targets mesothelin. ${ }^{56}$ The final RIT (LMB-T14) (Figure 1F) has good cytotoxic and anti-tumor activity versus human cell lines, patient-derived cells, and xenograft tumor models. To validate the reduced immunogenicity of LMB-T14, we compared the ability of LMB-T14 to bind to serum from patients treated with SS1P and remapped the T-cell epitopes using peptides from the mutated sequence of LMB-T14. We found that binding to human immunized sera was diminished with LMB-T14 compared with its parent molecules. Unfortunately, the mutations D463A and R467A, introduced to eliminate a conformational B-cell epitope, created a new T-cell epitope in peptide A457-W466. This demonstrates the complexity of balancing various mutations without compromising immunogenicity and cytotoxic activity.

\section{Immune Tolerance Induction}

The deimmunization efforts described above reduce, but do not eliminate, immunogenicity. To totally prevent immunogenicity, we have pursued a new strategy using synthetic vaccine particles encapsulating rapamycin (SVP-R) (Figure 3A).

Rapamycin is a mammalian target of rapamycin inhibitor that has both immunomodulatory properties and anti-tumor activity. ${ }^{58}$ Previous attempts to combine rapamycin with RITs were unsuccessful because the doses required to suppress the immune response were toxic to the mice. ${ }^{59}$ Encapsulation of rapamycin in SVP-R is effective in preventing ADA responses in mice against a variety of biological therapeutics, including adalimumab, PEGylated uricase, and coagulation factor VIII. ${ }^{60,61}$ Although the exact mechanism of preventing the immune response is not clear, the injection of the SVP-R results in its accumulation in the liver, spleen, and draining lymph nodes. ${ }^{62}$ Nanoparticles are preferentially phagocytosed by antigen-presenting cells because of their size, charge, and shape ${ }^{63}$ Selective delivery of rapamycin to these immune organs generates a tolerogenic milieu that can induce specific immune tolerance to coadministered antigens.

We evaluated SVP-R in combination with LMB-100, a RIT that is deimmunized from human B-cell epitopes but elicits ADA against both the human Fab and the toxin in mice. ${ }^{57}$ We found that SVP-R produces a long-lasting, specific, and transferable immune tolerance, which prevents $\mathrm{ADA}$ and neutralizing antibody formation against LMB-100 in naïve mice (Figure 3B). It is also effective in mice with preexisting antibodies (Figure $3 \mathrm{C}$ ). This immune tolerance resulted in restoration of the anti-tumor activity in an immunocompetent syngeneic mouse that would otherwise be neutralized by the ADA (Figure 3D).

\section{Concluding Remarks}

RITs have made their way from the bench to the bedside, where it was discovered that immunogenicity was a major obstacle in effectively treating patients with normal immune systems. To overcome this, we have now designed deimmunized RITs and developed a novel combination therapy to allow the deimmunized RITs to return to the bedside and be used for cancer treatment.

\section{Acknowledgment}

We thank Dr. Chin-Hsien Tai for the structural models in Figure 1.

\section{References}

1. Pastan I, Hassan R, Fitzgerald DJ, Kreitman RJ: Immunotoxin therapy of cancer. Nat Rev Cancer 2006, 6:559-565

2. Kreitman RJ, Tallman MS, Robak T, Coutre S, Wilson WH, StetlerStevenson M, Fitzgerald DJ, Lechleider R, Pastan I: Phase I trial of anti-CD22 recombinant immunotoxin moxetumomab pasudotox (CAT-8015 or HA22) in patients with hairy cell leukemia. J Clin Oncol 2012, 30:1822-1828

3. Kreitman RJ, Wilson WH, White JD, Stetler-Stevenson M, Jaffe ES, Giardina S, Waldmann TA, Pastan I: Phase I trial of recombinant immunotoxin anti-Tac(Fv)-PE38 (LMB-2) in patients with hematologic malignancies. J Clin Oncol 2000, 18:1622-1636

4. Mazor R, Onda M, Pastan I: Immunogenicity of therapeutic recombinant immunotoxins. Immunol Rev 2016, 270:152-164

5. Hassan R, Bullock S, Premkumar A, Kreitman RJ, Kindler H, Willingham MC, Pastan I: Phase I study of SS1P, a recombinant antimesothelin immunotoxin given as a bolus I.V. infusion to patients with mesothelin-expressing mesothelioma, ovarian, and pancreatic cancers. Clin Cancer Res 2007, 13:5144-5149

6. Khan DA: Hypersensitivity and immunologic reactions to biologics: opportunities for the allergist. Ann Allergy Asthma Immunol 2016, 117:115-120

7. Hassan R, Miller AC, Sharon E, Thomas A, Reynolds JC, Ling A, Kreitman RJ, Miettinen MM, Steinberg SM, Fowler DH, Pastan I: Major cancer regressions in mesothelioma after treatment with an anti-mesothelin immunotoxin and immune suppression. Sci Transl Med 2013, 5:208ra147

8. Hwang WY, Foote J: Immunogenicity of engineered antibodies. Methods 2005, 36:3-10

9. Harding FA, Stickler MM, Razo J, DuBridge RB: The immunogenicity of humanized and fully human antibodies: residual immunogenicity resides in the CDR regions. mAbs 2010, 2:256-265

10. Weldon JE, Pastan I: A guide to taming a toxin-recombinant immunotoxins constructed from Pseudomonas exotoxin A for the treatment of cancer. FEBS J 2011, 278:4683-4700

11. Pastan I: Immunotoxins containing Pseudomonas exotoxin A: a short history. Cancer Immunol Immunother 2003, 52:338-341

12. Weldon JE, Xiang L, Chertov O, Margulies I, Kreitman RJ, FitzGerald DJ, Pastan I: A protease-resistant immunotoxin against CD22 with greatly increased activity against CLL and diminished animal toxicity. Blood 2009, 113:3792-3800

13. Hansen JK, Weldon JE, Xiang L, Beers R, Onda M, Pastan I: A recombinant immunotoxin targeting CD22 with low immunogenicity, low nonspecific toxicity, and high antitumor activity in mice. J Immunother 2010, 33:297-304 
14. White TJ, Ibrahimi IM, Wilson AC: Evolutionary substitutions and the antigenic structure of globular proteins. Nature 1978, 274:92-94

15. Berzofsky JA: Intrinsic and extrinsic factors in protein antigenic structure. Science 1985, 229:932-940

16. Sathiamurthy M, Peters B, Bui HH, Sidney J, Mokili J, Wilson SS, Fleri W, McGuinness DL, Bourne PE, Sette A: An ontology for immune epitopes: application to the design of a broad scope database of immune reactivities. Immunome Res 2005, 1:2

17. Du L, Tai W, Yang Y, Zhao G, Zhu Q, Sun S, Liu C, Tao X, Tseng CK, Perlman S, Jiang S, Zhou Y, Li F: Introduction of neutralizing immunogenicity index to the rational design of MERS coronavirus subunit vaccines. Nat Commun 2016, 7:13473

18. Onda M, Nagata S, FitzGerald DJ, Beers R, Fisher RJ, Vincent JJ, Lee B, Nakamura M, Hwang J, Kreitman RJ, Hassan R, Pastan I: Characterization of the $\mathrm{B}$ cell epitopes associated with a truncated form of Pseudomonas exotoxin (PE38) used to make immunotoxins for the treatment of cancer patients. J Immunol 2006, 177: $8822-8834$

19. Liu W, Onda M, Lee B, Kreitman RJ, Hassan R, Xiang L, Pastan I: Recombinant immunotoxin engineered for low immunogenicity and antigenicity by identifying and silencing human B-cell epitopes. Proc Natl Acad Sci U S A 2012, 109:11782-11787

20. Onda M, Beers R, Xiang L, Nagata S, Wang QC, Pastan I: An immunotoxin with greatly reduced immunogenicity by identification and removal of B cell epitopes. Proc Natl Acad Sci U S A 2008, 105 : $11311-11316$

21. Onda M, Beers R, Xiang L, Lee B, Weldon JE, Kreitman RJ, Pastan I: Recombinant immunotoxin against B-cell malignancies with no immunogenicity in mice by removal of B-cell epitopes. Proc Natl Acad Sci U S A 2011, 108:5742-5747

22. Mazor R, Crown D, Addissie S, Jang Y, Kaplan G, Pastan I: Elimination of murine and human T-cell epitopes in recombinant immunotoxin eliminates neutralizing and anti-drug antibodies in vivo. Cell Mol Immunol 2017, 14:432-442

23. Alewine C, Hassan R, Pastan I: Advances in anticancer immunotoxin therapy. Oncologist 2015, 20:176-185

24. Bauss F, Lechmann M, Krippendorff BF, Staack R, Herting F, Festag M, Imhof-Jung S, Hesse F, Pompiati M, Kollmorgen G, da Silva Mateus Seidl R, Bossenmaier B, Lau W, Schantz C, Stracke JO, Brinkmann U, Onda M, Pastan I, Bosslet K, Niederfellner G: Characterization of a re-engineered, mesothelin-targeted Pseudomonas exotoxin fusion protein for lung cancer therapy. Mol Oncol 2016, 10: 1317-1329

25. Hollevoet K, Mason-Osann E, Liu XF, Imhof-Jung S, Niederfellner G, Pastan I: In vitro and in vivo activity of the lowimmunogenic antimesothelin immunotoxin RG7787 in pancreatic cancer. Mol Cancer Ther 2014, 13:2040-2049

26. Alewine C, Xiang L, Yamori T, Niederfellner G, Bosslet K, Pastan I: Efficacy of RG7787, a next-generation mesothelin-targeted immunotoxin, against triple-negative breast and gastric cancers. Mol Cancer Ther 2014, 13:2653-2661

27. Harding FA, Liu AD, Stickler M, Razo OJ, Chin R, Faravashi N, Viola W, Graycar T, Yeung VP, Aehle W, Meijer D, Wong S, Rashid MH, Valdes AM, Schellenberger V: A beta-lactamase with reduced immunogenicity for the targeted delivery of chemotherapeutics using antibody-directed enzyme prodrug therapy. Mol Cancer Ther 2005, 4:1791-1800

28. Cantor JR, Yoo TH, Dixit A, Iverson BL, Forsthuber TG, Georgiou G: Therapeutic enzyme deimmunization by combinatorial T-cell epitope removal using neutral drift. Proc Natl Acad Sci U S A 2011, 108:1272-1277

29. Cizeau J, Grenkow DM, Brown JG, Entwistle J, MacDonald GC: Engineering and biological characterization of VB6-845, an antiEpCAM immunotoxin containing a T-cell epitope-depleted variant of the plant toxin bouganin. J Immunother 2009, 32:574-584

30. Salvat RS, Choi Y, Bishop A, Bailey-Kellogg C, Griswold KE: Protein deimmunization via structure-based design enables efficient epitope deletion at high mutational loads. Biotechnol Bioeng 2015, 112:1306-1318

31. Tangri S, Mothe BR, Eisenbraun J, Sidney J, Southwood S, Briggs K, Zinckgraf J, Bilsel P, Newman M, Chesnut R, Licalsi C, Sette A: Rationally engineered therapeutic proteins with reduced immunogenicity. J Immunol 2005, 174:3187-3196

32. Yeung VP, Chang J, Miller J, Barnett C, Stickler M, Harding FA: Elimination of an immunodominant CD4 $+\mathrm{T}$ cell epitope in human IFN-beta does not result in an in vivo response directed at the subdominant epitope. J Immunol 2004, 172:6658-6665

33. Moise L, Song C, Martin WD, Tassone R, De Groot AS, Scott DW: Effect of HLA DR epitope de-immunization of factor VIII in vitro and in vivo. Clin Immunol 2012, 142:320-331

34. De Groot AS, Knopp PM, Martin W: De-immunization of therapeutic proteins by T-cell epitope modification. Dev Biol 2005, 122:171-194

35. Singh H, Raghava GP: ProPred: prediction of HLA-DR binding sites. Bioinformatics 2001, 17:1236-1237

36. Wang P, Sidney J, Kim Y, Sette A, Lund O, Nielsen M, Peters B: Peptide binding predictions for HLA DR, DP and DQ molecules. BMC Bioinformatics 2010, 11:568

37. Mazor R, Tai CH, Lee B, Pastan I: Poor correlation between T-cell activation assays and HLA-DR binding prediction algorithms in an immunogenic fragment of Pseudomonas exotoxin A. J Immunol Methods 2015, 425:10-20

38. Mazor R, Vassall AN, Eberle JA, Beers R, Weldon JE, Venzon DJ, Tsang KY, Benhar I, Pastan I: Identification and elimination of an immunodominant T-cell epitope in recombinant immunotoxins based on Pseudomonas exotoxin A. Proc Natl Acad Sci U S A 2012, 109: E3597-E3603

39. Mazor R, Eberle JA, Hu X, Vassall AN, Onda M, Beers R, Lee EC, Kreitman RJ, Lee B, Baker D, King C, Hassan R, Benhar I, Pastan I: Recombinant immunotoxin for cancer treatment with low immunogenicity by identification and silencing of human T-cell epitopes. Proc Natl Acad Sci U S A 2014, 111:8571-8576

40. Oseroff C, Sidney J, Kotturi MF, Kolla R, Alam R, Broide DH, Wasserman SI, Weiskopf D, McKinney DM, Chung JL, Petersen A, Grey H, Peters B, Sette A: Molecular determinants of T cell epitope recognition to the common Timothy grass allergen. J Immunol 2010 , 185:943-955

41. Stern LJ, Wiley DC: Antigenic peptide binding by class I and class II histocompatibility proteins. Structure 1994, 2:245-251

42. Koren E, De Groot AS, Jawa V, Beck KD, Boone T, Rivera D, Li L, Mytych D, Koscec M, Weeraratne D, Swanson S, Martin W: Clinical validation of the "in silico" prediction of immunogenicity of a human recombinant therapeutic protein. Clin Immunol 2007 , $124: 26-32$

43. Iwai LK, Yoshida M, Sidney J, Shikanai-Yasuda MA, Goldberg AC, Juliano MA, Hammer J, Juliano L, Sette A, Kalil J, Travassos LR, Cunha-Neto E: In silico prediction of peptides binding to multiple HLA-DR molecules accurately identifies immunodominant epitopes from gp43 of Paracoccidioides brasiliensis frequently recognized in primary peripheral blood mononuclear cell responses from sensitized individuals. Mol Med 2003, 9:209-219

44. Li X, Yang HW, Chen H, Wu J, Liu Y, Wei JF: In silico prediction of $\mathrm{T}$ and $\mathrm{B}$ cell epitopes of der $\mathrm{f} 25$ in dermatophagoides farinae. Int $\mathrm{J}$ Genomics 2014, 2014:483905

45. King C, Garza EN, Mazor R, Linehan JL, Pastan I, Pepper M, Baker D: Removing T-cell epitopes with computational protein design. Proc Natl Acad Sci U S A 2014, 111:8577-8582

46. Salvat RS, Parker AS, Guilliams A, Choi Y, Bailey-Kellogg C, Griswold KE: Computationally driven deletion of broadly distributed $\mathrm{T}$ cell epitopes in a biotherapeutic candidate. Cell Mol Life Sci 2014, 71:4869-4880

47. Ottenhoff TH, Elferink DG, Hermans J, de Vries RR: HLA class II restriction repertoire of antigen-specific T cells, I: the main restriction determinants for antigen presentation are associated with HLA-D/DR and not with DP and DQ. Hum Immunol 1985, 13:105-116 
48. Spurzem JR, Saltini C, Kirby M, Konishi K, Crystal RG: Expression of HLA class II genes in alveolar macrophages of patients with sarcoidosis. Am Rev Respir Dis 1989, 140:89-94

49. Meyer CG, May J, Schnittger L: HLA-DP-part of the concert. Immunol Today 1997, 18:58-61

50. Maurer DH, Hanke JH, Mickelson E, Rich RR, Pollack MS: Differential presentation of HLA-DR, DQ, and DP restriction elements by interferon-gamma-treated dermal fibroblasts. J Immunol 1987, 139:715-723

51. Mazor R, Addissie S, Jang Y, Tai CH, Rose J, Hakim F, Pastan I: Role of HLA-DP in the presentation of epitopes from the truncated bacterial PE38 immunotoxin. AAPS J 2017, 19:117-129

52. Mazor R, Zhang J, Xiang L, Addissie S, Awuah P, Beers R, Hassan R, Pastan I: Recombinant immunotoxin with T cell epitope mutations that greatly reduce immunogenicity for treatment of mesothelin expressing tumors. Mol Cancer Ther 2015, 14: $2789-2796$

53. Fleming BD, Nixon B, Pastan I, Ho M: Abstract 59: engineering glypican-3 targeting immunotoxins for the treatment of liver cancer. Cancer Res 2017, 77:59

54. Mazor R, Kaplan G, Park D, Jang Y, Lee F, Kreitman R, Pastan I: Rational design of low immunogenic anti CD25 recombinant immunotoxin for $\mathrm{T}$ cell malignancies by elimination of $\mathrm{T}$ cell epitopes in PE38. Cell Immunol 2017, 313:59-66

55. Kaplan G, Mazor R, Lee F, Jang Y, Leshem Y, Pastan I: Improving the in vivo efficacy of an anti-Tac (CD25) immunotoxin by Pseudomonas exotoxin A domain II engineering. Mol Cancer Ther 2018, $17: 1486-1493$

56. Mazor R, Onda M, Park D, Addissie S, Xiang L, Zhang J, Hassan R, Pastan I: Dual B- and T-cell de-immunization of recombinant immunotoxin targeting mesothelin with high cytotoxic activity. Oncotarget 2016, 7:29916-29926

57. Mazor R, King EM, Onda M, Cuburu N, Addissie S, Crown D, Liu XF, Kishimoto TK, Pastan I: Tolerogenic nanoparticles restore the antitumor activity of recombinant immunotoxins by mitigating immunogenicity. Proc Natl Acad Sci U S A 2018, 115:E733-E742

58. Stallone G, Infante B, Di Lorenzo A, Rascio F, Zaza G, Grandaliano G: mTOR inhibitors effects on regulatory T cells and on dendritic cells. J Transl Med 2016, 14:152

59. Mossoba ME, Onda M, Taylor J, Massey PR, Treadwell S, Sharon E, Hassan R, Pastan I, Fowler DH: Pentostatin plus cyclophosphamide safely and effectively prevents immunotoxin immunogenicity in murine hosts. Clin Cancer Res 2011, 17:3697-3705

60. Kishimoto TK, Ferrari JD, LaMothe RA, Kolte PN, Griset AP, O'Neil C, Chan V, Browning E, Chalishazar A, Kuhlman W, Fu FN, Viseux N, Altreuter DH, Johnston L, Maldonado RA: Improving the efficacy and safety of biologic drugs with tolerogenic nanoparticles. Nat Nanotechnol 2016, 11:890-899

61. Zhang AH, Rossi RJ, Yoon J, Wang H, Scott DW: Tolerogenic nanoparticles to induce immunologic tolerance: prevention and reversal of FVIII inhibitor formation. Cell Immunol 2016, 301:74-81

62. Maldonado RA, LaMothe RA, Ferrari JD, Zhang AH, Rossi RJ, Kolte PN, Griset AP, O'Neil C, Altreuter DH, Browning E, Johnston L, Farokhzad OC, Langer R, Scott DW, von Andrian UH, Kishimoto TK: Polymeric synthetic nanoparticles for the induction of antigen-specific immunological tolerance. Proc Natl Acad Sci U S A 2015, 112:E156-E165

63. Getts DR, Shea LD, Miller SD, King NJ: Harnessing nanoparticles for immune modulation. Trends Immunol 2015, 36:419-427 